\title{
Anomalous fluctuations of active polar filaments
}

\author{
Tanniemola B. Liverpool \\ Condensed Matter Theory Group, Blackett Laboratory, Imperial College, London SW7 2BZ, U.K. \\ Kavli Institute for Theoretical Physics, University of California, Santa Barbara, CA 93106
}

(November 20, 2018)

\begin{abstract}
Using a simple model, we study the fluctuating dynamics of inextensible, semiflexible polar filaments interacting with active and directed force generating centres such as molecular motors. Taking into account the fact that the activity occurs on time-scales comparable to the filament relaxation time, we obtain some unexpected differences between both the steady-state and dynamical behaviour of active as compared to passive filaments. For the statics, the filaments have a novel length-scale dependent rigidity. Dynamically, we find strongly enhanced anomalous diffusion.
\end{abstract}

Filamentous proteins are major components of the cell cytoskeleton [1]. Examples are actin filaments, microtubules and intermediate filaments. Their mechanical properties are important for cell stability and support and have been well studied at thermodynamic equilibrium by in vitro experiments. However, the conditions in the living cell are very different from those in the laboratory. Protein filaments interact with other proteins such as molecular motors and cross-linkers. This has led to a flurry of recent in vitro experiments of mixtures of filaments and their associated proteins in order to compare with their purified state [2, 4]. From a theoretical point of view, the proteins are typically far from equilibrium and therefore even to understand their steady-state behaviour, one has to study their dynamics. Non equilibrium effects have also recently been studied in biological membranes [5].

In this paper, motivated by recent experiments on Factin and myosin in the presence of ATP [ $⿴$, we study one example of the non-equilibrium behaviour of biofilaments: the fluctuating dynamics of polar filaments with active centres. This is also a model system for the study of non-trivial aspects of semiflexible filament dynamics. A key point of our analysis is the fact the activity of the proteins occurs over a time-scale $\tau$ which may be comparable to the relaxation time of the filament. Unlike recent work on motile solutions [6], the active centres considered here are associated with single filaments (i.e. are not cross-links) and cannot move one filament with respect to another. The viscosity of the solvent is given by $\eta$. We note that the typical energy scale of a biochemical reaction is of the order of a few $k_{B} T$ at physiological conditions.

The filament can be parameterised by a curve through its centre, $\mathbf{R}(s)$ (see Fig. 1). The unit tangent-vector is defined as $\mathbf{t}(s)=\partial \mathbf{R} / \partial s$. An easily measured quantity using e.g., video microscopy is the steady-state tan- gent correlation function $C_{\mathbf{t}}(s) \equiv\langle\mathbf{t}(s) \cdot \mathbf{t}(0)\rangle$. For a semiflexible polymer in equilibrium it decays exponentially, $C_{\mathbf{t}}(s)=\exp \left[-|s| / L_{p}\right]$ which defines the persistence length $L_{p}$.

Main results We calculate $C_{\mathbf{t}}(s)$ and find that due to activity on a time-scale $\tau$, the filaments develop a lengthscale dependent bending rigidity. On short length-scales, typical conformations have the bare persistence length whilst on longer length-scales the filaments may be characterised by a lower 'renormalized' persistence length. There is a cross-over length between the two regimes, $\ell_{c} \sim\left(\tau k_{B} T L_{p} / 2 \pi \eta\right)^{1 / 4}$. Therefore an analysis of the filament conformations can give information about the time-scale $\tau$ at which the activity occurs. We also obtain modified relaxational dynamics with anomalous diffusion which we show has an effect on the high frequency viscoelasticity. The shear modulus of the active filament solution apparently has a frequency dependent effective temperature. There is a crossover from at high frequencies a shear modulus corresponding to the bare temperature to at lower frequencies a modulus equivalent to the higher renormalized temperature both with a scaling of $\omega^{3 / 4}$. In the cross-over regime, the modulus appears to have a stronger frequency dependence with a power law $G^{*}(\omega) \propto \omega^{\alpha}$ where $\alpha>3 / 4$. A simple consequence of filament polarity is a small ballistic component to the motion of the filament,

Explicit analysis We model the filaments with the Kratky-Porod worm-like chain [7,8] which takes into account the bending energy cost of the chain. The worm-like chain Hamiltonian is $\mathcal{H}_{w l c}[\{\mathbf{R}(s)\}]=$ $\frac{\kappa}{2} \int_{-L / 2}^{L / 2} d s\left(\frac{\partial^{2} \mathbf{R}}{\partial s^{2}}\right)^{2}$. The stretching energy of chain molecules is much higher than the bending energy and we may consider the chain as inextensible. The persistence length is defined as $L_{p}=\kappa / k_{B} T$.

The dynamics at finite $T$ may be expressed by the Langevin equation

$$
\frac{\partial}{\partial t} \mathbf{R}(s, t)-\int d s^{\prime} \mathbf{H}\left(s, s^{\prime}\right) \cdot\left[-\kappa \frac{\partial^{4} \mathbf{R}}{\partial s^{\prime 4}}+\frac{\partial}{\partial s^{\prime}}\left(\Lambda\left(s^{\prime}, t\right) \frac{\partial \mathbf{R}}{\partial s^{\prime}}\right)\right]-\mathbf{V}(s, t)=\mathbf{f}(s, t)+\mathbf{f}^{(m)}(s, t),
$$


where $\Lambda(s, t)$ (a Lagrange multiplier) is an instantaneous, fluctuating 'tension' which enforces the local inextensibility of the chain. The inextensibility constraint fixes the tension, $\Lambda(s, t)$, by $0=\left|\frac{\partial \mathbf{R}}{\partial s}\right|^{2}-1$. Because of this constraint, the dynamics of semiflexible filaments is non-linear and generally insoluble. In addition to the thermal velocities $\mathbf{f}(s, t)$, there is an additional active velocities $\mathbf{f}^{(m)}(s, t)$ correlated over a time $\tau$. The polarity of the filament implies the active forces have a mean direction along the contour of the filaments (see Fig 1). Hydrodynamics is taken into account by the mobility tensor $\mathbf{H}\left(s, s^{\prime}\right)$. In the Stokes approximation valid for low Reynolds number, $\mathbf{H}\left(s, s^{\prime}\right)=$ $\mathbf{H}\left(\mathbf{R}(s)-\mathbf{R}\left(s^{\prime}\right)\right)$ and generates a non-dissipative term [9], $V_{i}(s, t)=k_{B} T \int_{0}^{L} d s^{\prime} \delta H_{i j}\left(s, s^{\prime}\right) / \delta R_{j}\left(s^{\prime}, t\right)$. In addition $\mathbf{f}(s, t)$ will also depend on $\mathbf{R}(s, t)$ [9]. We assume that the activity does not affect the thermal forces, i.e. the active proteins do not affect the collisions of the solvent molecules with the filament. Then the thermal velocities satisfy the fluctuation-dissipation theorem, and $\mathbf{f}(s, t)$ have zero mean and Gaussian fluctuations, given [9] by $\left\langle f_{i}(s, t) f_{j}\left(s^{\prime}, t^{\prime}\right)\right\rangle=2 k_{B} T H_{i j}\left(s, s^{\prime}\right) \delta\left(t-t^{\prime}\right)$.

We consider rod-like segments and restrict the analysis to length-scales $\ell$ below $L_{p}$ so that we can decompose the dynamics into transverse and longitudinal motion (see Fig. 11) and write the position of the filament as $\mathbf{R}(s, t)=\left(s-r_{\|}(s, t)\right) \hat{\mathbf{u}}(t)+\mathbf{r}_{\perp}(s, t)$ where $s \in$ $\{-\ell / 2, \ell / 2\}$ and $\hat{\mathbf{u}}$ is a time-dependent unit vector giving the orientation. We can obtain results within a systematic small gradient expansion for $\left|\mathbf{r}_{\perp}(s, t)\right|,\left|r_{\|}(s, t)\right| \ll$ $s$. The mobility tensor is given by $H_{i j}\left(s, s^{\prime}\right)=h(s-$ $\left.s^{\prime}\right)\left(\left(\delta_{i j}-\hat{u}_{i} \hat{u}_{j}\right)+2 \hat{u}_{i} \hat{u}_{j}+O\left(\left|\partial_{s} \mathbf{r}_{\perp}\right|^{2}\right)\right)$ where in this and the following, for a function $A(x), \partial_{x} A \equiv \partial A / \partial x$. We can thus decompose eqn. (1) into parallel and perpendicular components in an expansion to $O\left(\left|\partial_{s} \mathbf{r}_{\perp}\right|^{2}\right)$,

$$
\begin{aligned}
\partial_{t} \mathbf{r}_{\perp}(s, t) & =\int d s^{\prime} h\left(s-s^{\prime}\right)\left[-\kappa \partial_{s^{\prime}}^{4} \mathbf{r}_{\perp}+\Lambda\left(s^{\prime}, t\right) \partial_{s^{\prime}}^{2} \mathbf{r}_{\perp}+\partial_{s^{\prime}} \Lambda \partial_{s^{\prime}} \mathbf{r}_{\perp}\right]+\mathbf{f}_{\perp}(s, t)+\mathbf{f}_{\perp}^{(m)}(s, t) \\
\partial_{t} r_{\|}(s, t) & =\int d s^{\prime} 2 h\left(s-s^{\prime}\right)\left[-\kappa \partial_{s^{\prime}}^{4} r_{\|}-\partial_{s^{\prime}} \Lambda\right]+f_{\|}(s, t)+f_{\|}^{(m)}(s, t),
\end{aligned}
$$

which are coupled by the constraint of inextensibility,

$$
\partial_{s} r_{\|}=\frac{1}{2}\left(\partial_{s} \mathbf{r}_{\perp}\right)^{2}+O\left(\left|\partial_{s} \mathbf{r}_{\perp}\right|^{4}\right) .
$$

For simplicity in most of this paper, we consider the Rouse model which assumes local friction. We also focus on dilute solutions. Long range hydrodynamics and non-zero concentration of filaments will modify some of the results (see below). For the Rouse model $h\left(s-s^{\prime}\right)=\delta\left(s-s^{\prime}\right) / \zeta_{\perp}$ where $\zeta_{\perp}=4 \pi \eta=2 \zeta_{\|}$. We model the active velocities by a Gaussian noise with mean $\left\langle\mathbf{f}_{\perp}^{(m)}\right\rangle=0$ and $\left\langle f_{\|}^{(m)}\right\rangle=v_{m}$, a drift, reflecting the polar nature of the filament and mean square fluctuations, $\delta \mathbf{f}^{(m)}=\mathbf{f}^{(m)}-\left\langle\mathbf{f}^{(m)}\right\rangle$ given by

$$
\left\langle\delta f_{i}^{(m)}(s, t) \delta f_{j}^{(m)}\left(s^{\prime}, t^{\prime}\right)\right\rangle=\sqrt{2} \frac{\alpha_{i}}{\zeta_{i}^{2}} \Theta \delta_{i j} \delta\left(s-s^{\prime}\right) \Phi\left(t-t^{\prime}\right)
$$

where $\Phi(t)=\exp \{-|t| / \tau\}$ and $\{i, j\}$ refer to $\perp, \|$. The level of activity is controlled by the parameter $\Theta$. Correlations of the active force decay over a time, $\tau$ the typical activity period. The constants $\alpha_{i, j}$ measure the relative partitioning of the activity between transverse and longitudinal components and satisfy the relationship $\alpha_{\|}^{2}+\alpha_{\perp}^{2}=1$. In general, the force applied by the motor protein on the filament will not be purely tangential depending for example on its trajectory of approach of the filament and the conformation of the neck and chain region [1]. Averaging over orientations, only the longitudinal component will have a non-zero average, because of the filament polarity. In addition, the remainder of the energy of the actomyosin reaction that is not converted to directed work will be dissipated as heat contributing to the fluctuations on the same (reaction) time-scale. It is difficult apriori to estimate $\alpha_{i, j}$ and we choose somewhat arbitrarily, $\alpha_{\perp}=\alpha_{\|}=1 / \sqrt{2}$. Between active events the 'motors' diffuse freely in the solution so it is reasonable to assume that there is no spatial correlation between active sites. We emphasise that unlike the thermal noise, the active noise correlations do not satisfy the fluctuation dissipation theorem.

The eqns.(2, 3,, 4 ) are most easily studied by analysing the motion of bending modes of wave-vector $q$ and frequency $\omega$. Defining $F(s, t)=\int \frac{d q}{2 \pi} \frac{d \omega}{2 \pi} \tilde{F}(q, \omega) \exp (i \omega t+q s)$ we only consider $q$ such that $\pi / a \gg q \gg \pi / \ell$. On lengthscales below $L_{p}, C_{\mathbf{t}}(s)$ can be obtained from the transverse fluctuations. The transverse dynamics are approximately given by

$$
\tilde{\mathbf{r}}_{\perp}(q, \omega) \simeq \frac{\tilde{\mathbf{f}}_{\perp}(q, \omega)+\tilde{\mathbf{f}}_{\perp}^{(m)}(q, \omega)}{i \omega+\alpha q^{4}}
$$

where $\alpha=\kappa / \zeta_{\perp}$. Corrections due to the tension $\Lambda(s, t)$ are higher order in the gradient expansion. The transverse fluctuations at time $t$ are relaxed over a lengthscale $\ell_{\perp}(t)=(\alpha t)^{1 / 4}$. Because of inextensibility, eqn. (4) there is an induced time-independent longitudinal motion $r_{\|}^{(0)}(s)$ due to the averaged transverse motion, $\partial_{s} r_{\|}^{(0)}(s)=\left\langle\frac{1}{2}\left(\partial_{s} \mathbf{r}_{\perp}\right)^{2}\right\rangle_{\perp}$.

The tangent correlation function is given by 


$$
C_{\mathbf{t}}(s)=\langle\mathbf{t}(s, t) \cdot \mathbf{t}(0, t)\rangle \simeq\left\langle 1-\partial_{s} r_{\|}^{(0)}(s, t)-\partial_{s} r_{\|}^{(0)}(0, t)+\partial_{s} \mathbf{r}_{\perp}(s, t) \cdot \partial_{s} \mathbf{r}_{\perp}(0, t)\right\rangle_{\perp} .
$$

Using eqns. (4,6, 1, the tangent correlation function has the form

$$
C_{\mathbf{t}}(s) \simeq \exp \left\{-\frac{s}{L_{p}}-\frac{2 \Theta}{\alpha^{2}} \int \frac{d q}{2 \pi} \frac{1-\cos (q s)}{q^{2}\left(q^{4}+(\alpha \tau)^{-1}\right)}\right\} .
$$

The correlation function is plotted in Fig. 2. For lengthscales less than $\ell_{c} \simeq \ell_{\perp}(\tau)=\left(\kappa \tau / \zeta_{\perp}\right)^{1 / 4}$ the effective persistence length is approximately equal to the bare persistence length $L_{p}$ whilst for length-scales above $\ell_{c}$, the conformations can be well modelled by a wormlike chain with a lower renormalized persistence length given by $L_{p}^{*}=L_{p}\left(1+\Theta \tau \zeta_{\perp} / k_{B} T\right)^{-1}$. Dimensional analysis suggests a new active temperature scale given by $k_{B} T_{a}=\Theta \tau \zeta_{\perp}$.

Dynamics The dynamics of inextensible filaments is anisotropic [10, 11, 13, 14]: longitudinal motion has different relaxation dynamics to the transverse. Viscous dissipation due to longitudinal motion must be taken into account, giving a time-scale over which 'tension' propagates along the filament [10,11,13,14. From eqn. (6), we can calculate the transverse dynamic fluctuations, $R_{\perp}^{2}(t)=\left\langle\left(\mathbf{r}_{\perp}(s, t)-\mathbf{r}_{\perp}(s, 0)\right)^{2}\right\rangle_{\perp}$

$$
R_{\perp}^{2}(t)= \begin{cases}t^{2} \frac{\Theta}{\sqrt{2}} \kappa^{3 / 4} \zeta_{\perp}^{-3 / 4} \tau^{7 / 4}, & t \ll \tau \\ t^{3 / 4} \frac{8 \Gamma(1 / 4)}{3 \pi} \kappa^{1 / 4} \zeta_{\perp}^{-3 / 4}\left(k_{B} T+\Theta \zeta_{\perp} \tau\right), & t \gg \tau\end{cases}
$$

There is a cross-over from ballistic motion at short times to sub-diffusive behaviour at long times. Note the thermal and active contributions to the sub-diffusive regime.

We can solve for the longitudinal motion selfconsistently as follows: we average over only the transverse fluctuations and use the inextensibility constraint 13.14 to give a relationship between the tension and the longitudinal motion, $i q\left(\tilde{r}_{\|}(q, \omega)-\tilde{r}_{\|}^{(0)}(q)\right)=$ $K(\omega) \tilde{\Lambda}(q, \omega)$ defining a frequency dependent extensional compliance, $K(\omega)=K_{e q}(\omega)+K_{a}(\omega)$ which has equilibrium and active contributions. The equilibrium modulus is given by $K_{e q}(\omega) \simeq 2^{-3 / 4} k_{B} T \kappa^{-5 / 4}\left(i \zeta_{\perp} \omega\right)^{-3 / 4}$ is not new and has been obtained previously [11,12]. We find in addition a new active contribution given by

$$
K_{a}(\omega)=\frac{4 \Theta \tau}{\kappa} \int_{k} \frac{\left(\alpha k^{4} \tau+f\left(\alpha k^{4} \tau\right)-i \omega \tau\right)}{f\left(\alpha k^{4} \tau\right)\left(2 \alpha k^{4}-i \omega\right)\left(f\left(\alpha k^{4} \tau\right)-i \omega \tau\right)}
$$

where $f(x)=x+1$. In the limit $\omega \rightarrow 0$, it is given by $K_{a}(\omega) \simeq 2^{-3 / 4} \Theta \tau \zeta_{\perp} \kappa^{-5 / 4}\left[\left(i \omega \zeta_{\perp}\right)^{-3 / 4}-\frac{1}{4}\left(\zeta_{\perp} / 2 \tau\right)^{-3 / 4}\right]+$ $O(\omega)$. The modulus $K(\omega)$ is then substituted into the longitudinal dynamics. This self-consistent approach corresponds to an infinite resummation of a set of diagrams of the perturbation expansion in $\Lambda$ [13]. We obtain the following equation for the longitudinal motion,

$$
\tilde{r}_{\|}(q, \omega)-\tilde{r}_{\|}^{(0)}(q) \simeq \frac{\tilde{f}_{\|}(q, \omega)+\tilde{f}_{\|}^{(m)}(q, \omega)}{i \omega+q^{2} K^{-1}(\omega) / \zeta_{\|}}
$$

As defined above, $r_{\|}^{(0)}(s)$ is the time-independent motion in the longitudinal direction due to the averaged transverse motion. Given this, we calculate the longitudinal dynamical fluctuations, $R_{\|}^{2}(t)=\left\langle\left(r_{\|}(s, t)-r_{\|}(s, 0)\right)^{2}\right\rangle_{\|}$

$$
R_{\|}^{2}(t)= \begin{cases}t^{2}\left(v_{m}^{2}+\Theta \zeta_{\|}^{1 / 2} 2^{-3 / 4} \kappa^{3 / 8} \zeta_{\perp}^{-3 / 8} \tau^{1 / 8}\right), & t \ll \tau \\ t^{2} v_{m}^{2}+t^{7 / 8} \frac{8 \Gamma(1 / 8)}{7 \pi} \frac{(2 \kappa)^{5 / 8}\left(k_{B} T+\Theta \zeta_{\perp} \tau\right)^{3 / 8}}{\zeta_{\|}^{7 / 8}} t \gg \tau .\end{cases}
$$

Because of the drift term (the directed motor/filament interaction), the longitudinal diffusion is ballistic for all time-scales.

High frequency viscoelasticity The complex shear modulus of a solution of semiflexible filaments at high frequencies is dominated by the extensional compliance and $G^{*}(\omega)=\frac{2}{15} \rho K^{-1}(\omega) \simeq$ $0.133 \rho /\left(K_{e q}(\omega)+K_{a}(\omega)\right)$ [6,11, 12 which for a solution of passive filaments is given by $G^{*}(\omega) \propto \omega^{3 / 4}$. For the active filaments, using eqn. (10), we plot the absolute value of the complex high frequency modulus for varying activity in Fig. 3. We see a crossover from at high frequencies a modulus corresponding to the bare temperature, $T$ to at low frequencies a modulus equivalent to the higher renormalized active temperature $T+T_{a}$, both with a scaling of $\omega^{3 / 4}$. In the cross-over regime, the modulus appears to have a stronger frequency dependence with a power law $G^{*}(\omega) \propto \omega^{\alpha}$ where $\alpha>3 / 4$. It is interesting to note that the cross-over occurs over a very wide frequency range.

Hydrodynamics Within the (screened) Oseen approximation the mobility tensor is given by [7], $H_{i j}[\mathbf{r}]=$ $\frac{e^{-|\mathbf{r}| / \xi}}{8 \pi \eta|\mathbf{r}|}\left(\delta_{i j}+\hat{r}_{i} \hat{r}_{j}\right)$ for $|\mathbf{r}|>a$. The hydrodynamic screening length $\xi \rightarrow \infty$ for dilute solutions and is equal to the mesh-size $\xi=\left(\rho_{a} a\right)^{-1 / 2}$ for semi-dilute solutions with actin concentration $\rho_{a}$ and filament diameter $a . . \quad$ Therefore, $h\left(s-s^{\prime}\right)=\frac{e^{-\left|s-s^{\prime}\right| / \xi}}{8 \pi \eta\left|s-s^{\prime}\right|}$ in eqns (2, 33). For dilute solutions $(\xi \rightarrow \infty)$, this gives logarithmic corrections to the Rouse model described above and implies a modification of the cross-over length to $\ell_{c} \sim\left(\kappa \tau / \zeta_{\perp} \log \left[\left(\kappa \tau / \zeta_{\perp}\right)^{1 / 4} / a\right]\right)^{1 / 4}$. For concentrated solutions ( $\xi$ finite), the friction coefficients cross-over length is $\ell_{c} \sim\left(\kappa \tau / \zeta_{\perp} \log [\xi / a]\right)^{1 / 4}$. 
Entangled solutions For entangled solutions, the filament can be modelled as confined in its tube. Given a mesh-size $\xi$, we can define a tube diameter $D_{e} \sim L_{p}\left(\xi / L_{p}\right)^{6 / 5}$ and entanglement length $L_{e} \sim$ $L_{p}\left(\xi / L_{p}\right)^{4 / 5}$ [15]. The tube can be modelled as a confining potential for $\mathbf{r}_{\perp}$ which we can model as $V_{\text {tube }}=$ $\frac{1}{2} k\left|\mathbf{r}_{\perp}\right|^{2}$ with $k \simeq \kappa / L_{e}^{4}$ chosen so as to give \langle| $\mathbf{r}_{\perp}\left(L_{e}\right)-$ $\left.\left.\mathbf{r}_{\perp}(0)\right|^{2}\right\rangle^{1 / 2}=D_{e}$, the tube diameter. We obtain the tangent correlation function, $C_{\mathbf{t}}(s)=1-\frac{s}{L_{p}}-$ $\frac{2 \Theta}{\alpha^{2}} \int \frac{d q}{2 \pi} \frac{1-\cos (q s)}{q^{2}\left(q^{4}+(\alpha \tau)^{-1}+k / \kappa\right)}$.

Discussion Our results should be relevant for recent experiments on mixtures of $\mathrm{F}$-actin and S1 Myosin [ 4 ]. The molecular motor myosin interacts with F-actin in the presence of ATP undergoing a conformational change in the process. Hydrophobic interactions between tails of a common variant, Myosin II, lead to multi-headed clusters which can act as active cross-links [3, 6] between two or more filaments. In contrast, S1 myosin is a single-headed version of myosin that is without a tail. Therefore, in general S1 myosin/ATP interacts with single polar actin filaments applying biased non-equilibrium forces. The S1 experiments found surprisingly different steady-state and dynamic behaviour of the filaments as compared to the pure F-actin system [4].

We now estimate the values for the parameters of our model corresponding to the S1 experiment. For an actin monomer concentration $\rho_{a}$, the fraction of bound myosin $\phi$ can be estimated using the equilibrium constant [1], $k_{e q}=500 \mathrm{nM}$ for the passive reaction (Actin + Myosin $\Leftrightarrow$ Actomyosin). The fraction of bound myosin is given by $\phi=\rho_{a} /\left(k_{e q}+\rho_{a}\right)$ leading to the typical separation of the active sites (motors) on the filament, $\ell_{m} \simeq\left(\phi \rho_{m} \xi^{2}\right)^{-1}=$ $a\left(\rho_{a} / \phi \rho_{m}\right)$ where $\rho_{m}$ is the concentration of S1 myosin. Then we may divide the filament into regions of size $\ell_{m}$ in which the motors are expected to act independently. Therefore $\left\langle\mathbf{f}(s, t) \mathbf{f}\left(s^{\prime}, t^{\prime}\right)\right\rangle=\ell_{m} \delta\left(s-s^{\prime}\right)\left\langle\mathbf{f}_{m}(t) \mathbf{f}_{m}\left(t^{\prime}\right)\right\rangle$. We assume that motor attachment on the filaments is a Poisson process [17], i.e. that motors arrive at random times $t_{n}$ with a constant rate $\lambda$. The number of motors arriving in a period $\Delta t \gg 1 / \lambda$ has a Poisson distribution. The forces applied by each motor are assumed to decay over a timescale $\tau$ so that the force at time $t$ is given by

$$
\mathbf{F}_{m}(t)=\sum_{n=1} \frac{a}{\ell_{m}} \mathbf{f}_{0} g\left(t-t_{n}\right)
$$

where $g(t)=\exp (-t / \tau), t>0$ and $g(t)=0, t<0$ and $\mathbf{f}_{0}$ is the typical force applied by a motor to a filament of diameter $a$. After some standard manipulations [17] and averaging over orientations, the velocity correlations can be shown to be given by eqn. (5) above with the activity parameter given by $\Theta \simeq \lambda \tau \ell_{m}^{-1}\left(f_{0} a\right)^{2}$ and we set $\lambda=1 / \tau$. Similarly, the drift can be estimated as $v_{m} \simeq\left(f_{0} / \zeta_{\|}\right) \ell_{m}^{-1}$. The typical force, $f_{0}$ and active time-scale, $\tau$ can be obtained from biochemical data. The viscosity of water is $\eta \simeq 10^{-3} \mathrm{~Pa} \mathrm{~s}$ and $\mathrm{F}$-actin has diameter $a \simeq 7 \mathrm{~nm}$ and persistence length $L_{p} \simeq 15 \mu \mathrm{m}$. The stall force of Myosin is approximately $5 \mathrm{pN}$ and the duration of the motor cycle is approximately $\tau \simeq 5 \mathrm{~ms}$ The motor step-size $d_{m}$ is approximately $d_{m} \simeq 10 \mathrm{~nm}$. For ATP saturation and $5 \mu \mathrm{M}$ $\mathrm{S} 1$ and $14 \mu \mathrm{M} \mathrm{F}$-actin concentrations, we find $\phi \approx 0.9$ and $\ell_{m} \approx 2 a$. This leads to an estimate for $\Theta \zeta_{\perp} \tau \simeq k_{B} T$ at $310 \mathrm{~K}$ giving $L_{p}^{*} \simeq \frac{1}{2} L_{p}$ and $\ell_{c} \sim 0.1 \mu \mathrm{m}$.

In conclusion, we have studied a simple model of active filaments and obtained different static and dynamical properties as compared to passive semiflexible polymers. These differences should be observable with video microscopy and in linear rheological experiments and be relevant for experiments on actin-myosin systems.

We thank A. Ajdari, F. Amblard, E. Furst, D. Humphrey, F. Jülicher, J. Käs, L. Le Goff and A.C. Maggs for discussions. The financial support of the Royal Society and the National Science Foundation under grants PHY-99-07949 (at KITP) is gratefully acknowledged .

[1] J. Howard, Mechanics of Motor Proteins and the Cytoskeleton, (Sinauer, New York, 2000).

[2] F. Nedelec et al. Nature 389, 305, (1997).

[3] D. Humphrey, C. Duggan, D. Saha, D. Smith and J. Käs, Nature 416, 413, (2002).

[4] L. Le Goff, F. Amblard and E. Furst, Phys. Rev. Lett. 88, 018101, (2002).

[5] J. Prost and R. Bruinsma, Europhys. Lett. 33, 321 (1996); J. Prost, J.-B. Manneville and R. Bruinsma, Eur. Phys. J. B, 1, 465 (1998); R. Granek and S. Pierrat, Phys. Rev. Lett. 83, 875, (1999).

[6] T. B. Liverpool, A. C. Maggs and A. Ajdari Phys. Rev. Lett. 86, 4171, (2001).

[7] M. Doi and S.F. Edwards, The Theory of Polymer Dynamics, (Clarendon, Oxford, 1992).

[8] H. Isambert and A.C. Maggs, Macromolecules 29, 1036 (1996).

[9] Chaikin, P.; Lubensky, T. Principles of Condensed Matter Physics, C.U.P.: Cambridge, U.K., 1995.

[10] R. Everaers et al, Phys. Rev. Lett. 82, 3717, (1999).

[11] D.C. Morse, Macromolecules 31, 7030 (1998); Macromolecules 31, 7044 (1998); Phys. Rev. E. 58, R1237 (1998).

[12] F. Gittes and F.C. MacKintosh, Phys. Rev. E. 58, R1241 (1998).

[13] T.B. Liverpool and A.C. Maggs, Macromolecules 34, 6064-6073 (2001).

[14] M. Pasquali, V. Shankar, and D. C. Morse Phys. Rev. E. 64, 020802(R) (2001).

[15] T. Odijk, Macromolecules 16, 1340, (1983); M. Doi, J. Polym. Sci.: Polym. Symp. 73, 93, (1985); A.N. Semenov, J. Chem. Soc., Faraday Trans. 2, 317, (1986).

[16] R.D. Vale and R.A. Milligan, Science 288, 88 (2000); C. Viegel et al, Biophys. J. 75, 1424 (1998). 
[17] C.W. Gardiner, Handbook of Stochastic Processes, (Springer, Berlin , 1985).

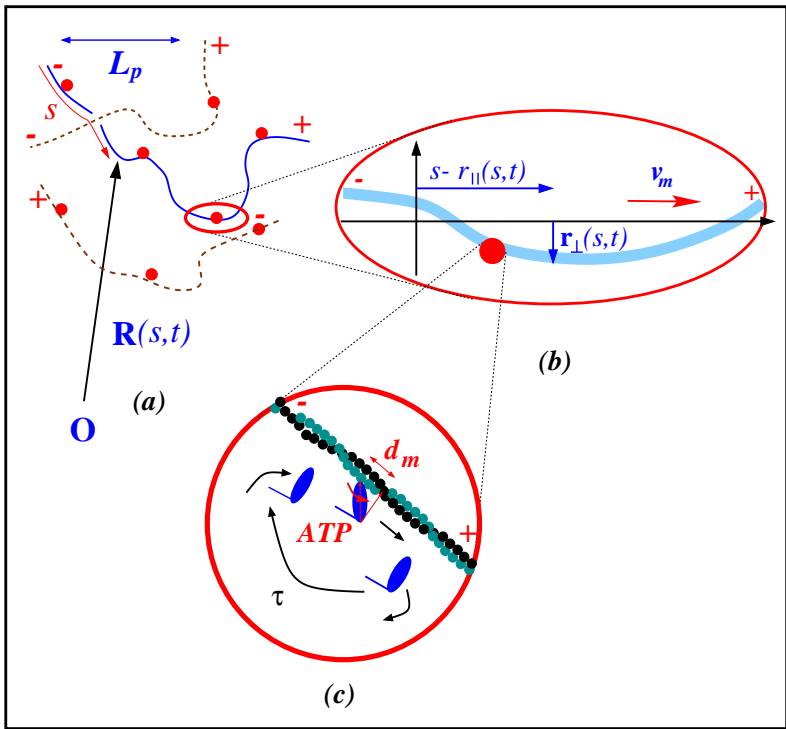

FIG. 1. (a) The fluctuating polar filaments of persistence length $L_{p}$ parametrised by $\mathbf{R}(s, t)$ decorated by active centres. Note that there is a $(+)$ and a $(-)$ end for each filament. (b) The filament on length-scales below $L_{p}$ showing the transverse $\mathbf{r}_{\perp}(s, t)$ and longitudinal $r_{\|}(s, t)$ motion. (c) A schematic of the cycle of activity of a motor such as myosin with activity time $\tau$.

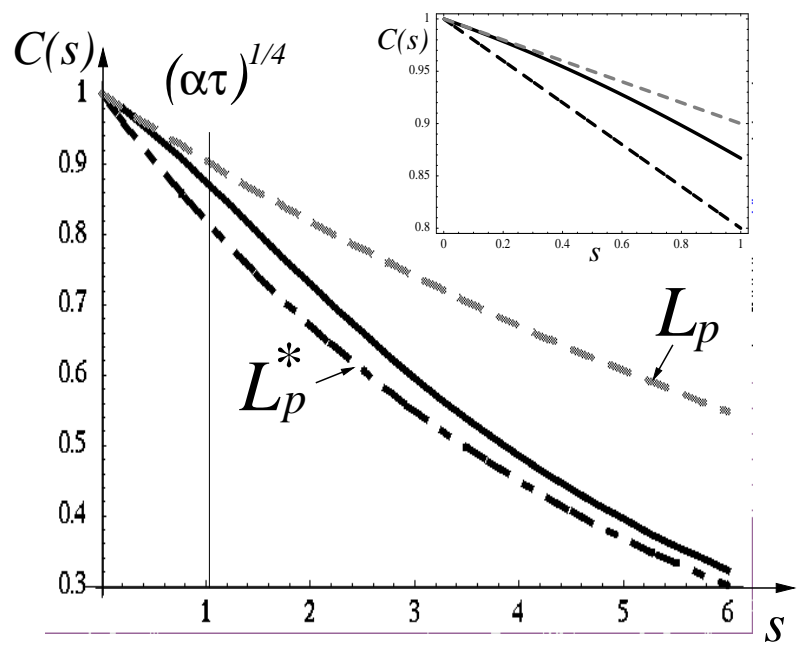

FIG. 2. The tangent correlation function for the active filament of bare persistence length $L_{p}=10$, compared with those simple wormlike chains of persistence length of $L_{p}=10$ and $L_{p}^{*}=5$ using units such that $\alpha \tau=1$. Inset: $C_{\mathbf{t}}(s)$ near $s=0$.

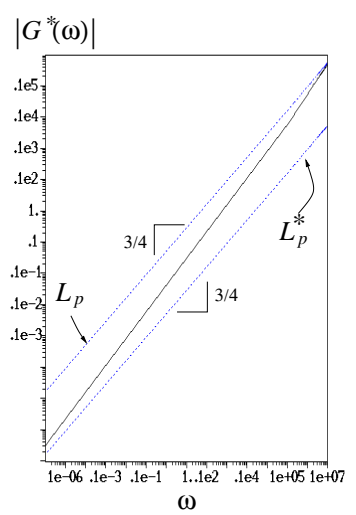

(a)

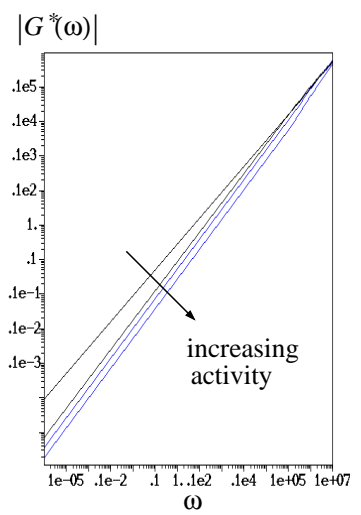

(b)
FIG. 3. The magnitude of the high frequency modulus for active filaments. We have chosen units of length and time such that $\alpha \tau=1$ and $k_{B} T / \zeta_{\perp}=1$. In these units, the bare persistence length of the filaments is $L_{p}=1$. (a) The cross-over to a renormalised persistence length $L_{p}^{*}=1 / 101$. (b) Increasing activity corresponding to $\Theta \zeta_{\perp} \tau=k_{B} T_{a}=25,50,100$ 